\title{
BMJ Open How do community advisory boards fulfil their ethical role in HIV clinical trials? A protocol for a systematic review of qualitative evidence
}

\author{
Godwin Pancras (D) , ${ }^{1}$ Maryam Amour, ${ }^{2}$ Tosi Mwakyandile, ${ }^{3}$ Baraka Morris, ${ }^{4}$ \\ Bruno F Sunguya, ${ }^{2}$ Blandina Mmbaga ${ }^{5}$
}

To cite: Pancras G, Amour M, Mwakyandile T, et al. How do community advisory boards fulfil their ethical role in HIV clinical trials? A protocol for a systematic review of qualitative evidence. BMJ Open 2020;10:e035368. doi:10.1136/ bmjopen-2019-035368

- Prepublication history for this paper is available online. To view these files, please visit the journal online (http://dx.doi org/10.1136/bmjopen-2019035368).

Received 29 0ctober 2019 Revised 07 March 2020 Accepted 16 April 2020
Check for updates

(C) Author(s) (or their employer(s)) 2020. Re-use permitted under CC BY-NC. No commercial re-use. See rights and permissions. Published by BMJ.

For numbered affiliations see end of article.

Correspondence to

Godwin Pancras;

katunzip@gmail.com

\section{ABSTRACT}

Introduction Community advisory boards (CABs)

continue to gain wide use and acceptance in global health research including in HIV clinical trials. They provide means through which community concerns regarding the trial can be considered by the research team, and provide an important platform of communication between the researchers and the community about study goals. Therefore, this systematic review protocol will guide the review of qualitative evidence on the ethical roles of CABs in HIV clinical trials based on the three fundamental ethical principles: respect for the person, beneficence and justice. Methods and analysis This systematic review of qualitative evidence will involve searching four medical databases: PubMed, ScienceDirect, CINAHL and Cochrane Library. Additionally, other relevant evidence will be obtained through hand searching and grey literature. Searches will be limited to studies published in the English language from 1989 (the year that CABs were first established in HIV clinical trials) to 2019. Articles searched will be screened by two independent authors based on inclusion and exclusion criteria. Included articles will be appraised for quality using the Critical Appraisal Skills Programme checklist and followed by qualitative data extraction. Findings will be analysed based on the metaaggregative approach with the aid of EPPI-Reviewer 4 web-based software.

Ethics and dissemination Ethical approval does not apply to this review. Data will be disseminated through scientific conferences and peer-reviewed journals to inform policies and stake-holders about the ethical role of CABs.

PROSPERO registration number CRD42019133787.

\section{INTRODUCTION}

Since the 1980s, community advisory boards (CABs) have been devised as prominent mechanisms for researchers to effectively engage communities being researched. ${ }^{1}$ They continue to gain wide use and acceptance in global health research including in HIV clinical trials. The $\mathrm{CAB}$ provides the pipeline through which community concerns can be aired-out to the research team, but also, a
Strengths and limitations of this study

- The study will systematically review qualitative data from various medical databases and grey literature for an in-depth understanding of the ethical roles of community advisory boards in HIV clinical trials.

- All articles included in the final review will be appraised for quality using the Critical Appraisal Skills Programme for qualitative studies.

- Data management and extraction will be aided by web-based software called EPPI-Reviewer 4.

- The meta-aggregation approach used will only permit the synthesis of text data.

- The meta-aggregation approach used will not seek to reinterpret primary findings from the included studies.

means for the research team to communicate with the community. ${ }^{2}$ Depending on member composition, CABs have been divided into two models: population-specific model and broad community model. ${ }^{3}$ Members of the former model involve potential research participants or people with first-hand experience of the disease or phenomenon being studied. For example, the CAB for an HIV clinical trial would be composed of people living with HIV, people who lost their loved ones to the disease and hospital-based healthcare providers caring for people living with HIV. On the other hand, the broad community model is composed of a wide spectrum of community representatives from politicians, educators, vulnerable population representatives to community and religious leaders. ${ }^{4}$ Anecdotally, the broad community model is less expensive compared with populationspecific model since members do not have to be reshuffled based on a research protocol.

Some scholars have regarded but yet questioned the ethical role of CABs as the gatekeeper to community and individual 
participants' interests. ${ }^{5}$ This questioning is further complicated by the fact that CABs also have another role of advancing the goals of research. Much of the literature available are single studies focused on the history, composition and function of specific CABs at a particular HIV research site. ${ }^{2367}$ Hence, with the paucity and individualised information regarding the role of CABs, a systematic review is warranted to identify, organise and critically appraise such studies and understand the ethical role of CABs in HIV clinical trials. This will help policymakers, researchers and funders make an informed decision regarding what ought to be the role of CABs in an HIV clinical trial. Consequently, this systematic review will serve as a point of reference for future trials (including non-HIV related studies) seeking to establish CABs. We focus on HIV clinical trials because HIV remains one of the global public health challenges whereby community involvement mechanisms in research (like using CABs) are crucial in the fight against the virus.

The framework for exploring the ethical role of CABs in HIV clinical trials will be inductively centred on the fundamental ethical principles which emanate from the Belmont report. ${ }^{8}$ Whereby the first principle calls on protecting and enhancing individual participant autonomy (the principle of respect for persons). The second principle calls on reduction of harm and striving to maximise benefits of any given trial (the principle of beneficence). Lastly, the third principle calls for fairness in the selection of participants and distribution of burdens and benefits of a trial (the principle of justice). Thus, no research conducted beyond the three principles could be regarded as ethical. ${ }^{9}$ However, it should be noted that for this study the principles are extended to include other aspects of research and the community at large through an inductive approach.

\section{Aim}

The protocol aims to review qualitative evidence regarding the ethical role of CABs in HIV clinical trials through their involvement in implementing the three fundamental ethical principles. The systematic review will involve identifying, appraising and synthesising qualitative studies that have examined CAB involvement in informed consent process (principle of respect for person), riskbenefit analysis (beneficence) and trial participant selection (justice).

\section{METHODS AND ANALYSIS}

The study was initiated in May 2019 and we expect it to be completed by February 2020.

\section{Search strategy}

We will first develop the following domains based on the review question: role, community, HIV, advisory, board and clinical trial. From each domain, we will create alternative terms which vary based on the database being searched. We will modify the search strategy for each database depending on the user interphase and number of terms allowed. Example, we will use the following search term in PubMed: (( ( ((Role*) OR Responsibility*) OR Function*) $)$ AND $(((($ Clinical trial*) OR Clinical research*) OR Study*) OR Research*)) AND (((HIV) OR AIDs) OR Human immunodeficiency virus)) AND (( (community) AND advisory) AND board*)).

\section{Data sources}

The following bibliographic databases will be searched: PubMed, ScienceDirect, CINAHL and Cochrane Library. Also, we will hand search for references of some papers of interest and other grey literature.

\section{Eligibility criteria for the studies}

Inclusion criteria

Searches will be limited to studies published in the English language from 1989 (the year that CABs were first deployed in HIV clinical trials) to 2019. Studies using qualitative approaches such as focus group discussion, in-depth interview, document review and any written conversations will be explored. We will also include studies conducted using mixed methods approaches, but only the qualitative findings will be considered.

\section{Exclusion criteria}

Studies documenting utilisation of CABs in trials other than HIV trials. Also, studies using a quantitative approach (except for open-ended questions) will be excluded from the analysis. The same applies to articles without full text.

\section{Data management}

Reference from bibliographic databases will be managed by Zotero Standalone V.5.0.69. Then, full-text articles retrieved after review will be uploaded to EPPI-Reviewer 4 web-based software for qualitative data extraction and synthesis. ${ }^{10}$ A Preferred Reporting Items for Systematic Reviews and Meta-Analyses statement flow diagram will be used to illustrate how studies have been identified, screened and included in the final review. ${ }^{11}$

\section{Selection of studies}

The titles or/and abstracts of studies retrieved using the above search strategy will be screened independently by two researchers from the review team who have been blinded from each other's decision. Studies meeting the inclusion criteria will be subject to full-text review by the same researchers independently. After which, the team will meet up for discussion and resolving any disagreements that may arise about which studies should be included.

\section{Data extraction}

The EPPI-Reviewer 4 web-based software will be used for data management and extraction of the qualitative data gathered. ${ }^{10}$ Data extracted from included studies will include study aim/objective, study design, data collection method, study participants, data analysis and study findings related to the ethical role for CABs in HIV clinical 
trials. The process for data extraction will be carried out independently by two researchers of the review team. Findings from the included articles will include themes, subthemes, direct words from the authors and actual participant quotes. Discrepancies between two researchers will be resolved through discussion, but in case an issue in question cannot be easily resolved, a third independent researcher will be sought for advice.

\section{Risk of bias (quality) assessment}

The risk of bias and quality of potential studies and other documents will be independently assessed by two reviewers. The Critical Appraisal Skills Programme (CASP) checklist will be used to identify the quality of qualitative studies to be included in the review. ${ }^{12}$ Discrepancies will be resolved through discussions with the review team. The CASP qualitative checklist consists of 10 questions: (1) Was there a clear statement of the aims of the research? (2) Is a qualitative methodology appropriate? (3) Was the research design appropriate to address the aims of the research? (4) Was the recruitment strategy appropriate to the aims of the research? (5) Were the data collected in a way that addressed the research issue? (6) Has the relationship between the researcher and participants been adequately considered? (7) Have ethical issues been taken into consideration? (8) Was the data analysis sufficiently rigorous? (9) Is there a clear statement of findings? (10) How valuable is the research? It is important to note that the tool will not be used to make decisions whether to include or exclude studies but rather to identify the strength of included studies.

\section{Strategy for data synthesis}

Findings from selected studies will be synthesised according to the Joanna Briggs Institute (JBI) metaaggregation three-step process, ${ }^{13}$ which includes: (1) extraction of findings or categories and quotes and illustrations from primary studies; (2) synthesis of findings into categories based on their similarities and lastly (3) synthesis of identified categories into a set of synthesised findings (or analytical themes). But to avoid newly formulated categories being less informative or overlapping, full sentences will be used to present the categories generated by meta-aggregation. The whole process of data synthesis will deploy both inductive and deductive qualitative analysis techniques. All steps will be conducted by two independent researchers and their findings will be clustered together to generate informative synthesised findings.

\section{Patient and public involvement}

No patient will be involved in the development of the systematic review. However, we consulted experts of systematic reviews and clinical trials for their inputs. Most indicated that the study will be useful given that nearly every clinical trial is required to demonstrate $\mathrm{CAB}$ involvement during grant application.

\section{DISCUSSION}

This manuscript describes a protocol for a systematic review of qualitative evidence on the ethical role of CABs in HIV clinical trials. The strength of which relies on the use of systematic procedures to identify and analyse non-numerical information gathered through qualitative techniques. Although the use of qualitative evidence to conduct systematic reviews is hotly contested, the increased need to inform policies and practices in detail has pressed it on high demand. ${ }^{14}{ }^{15}$ For this systematic review, qualitative evidence will be systematically identified, screened, appraised and synthesised to develop analytical themes depicting the ethical role of CABs in HIV clinical trial. Studies using non-qualitative techniques to collect primary information will be excluded. For each stage of the review, at least one reviewer will be involved and discrepancies which cannot be resolved through discussion within the research team, an independent expert researcher will be consulted.

The role of CABs to be termed as ethical, a reflection is made to the fundamental ethical principles portrayed in the Belmont report. ${ }^{8}$ These principles include: respect for persons (involving the informed consent process), beneficence (involving weighing risks/benefits) and justice (involving a fair participant selection). The principles have also acted as the backbone for most international ethical guidelines including the international ethical guidelines for health-related research involving humans and the Declaration of Helsinki. ${ }^{16}{ }^{17}$ Although the principles have existed for over three decades, the understanding of how CABs in HIV clinical trials are grappling with them is limited. Moreover, no systematic reviews currently exist to address this phenomenon. For preliminary searches we have conducted, several individual studies exist, but most being site and population specific. Nonetheless, these studies outline a plethora of perspectives on what was the role of $\mathrm{CAB}$ members in the HIV clinical trial at a given study site. ${ }^{67}$

Therefore, this systematic review will inform policymakers, researchers and funders of HIV clinical trials on key ethical issues to consider when working with, or establishing CABs. More importantly, the review will shade a light to the international research community on whether CABs provide a reliable mechanism for protecting the host community or participants' interests.

\section{ETHICS AND DISSEMINATION}

The systematic review will be disseminated through publication in peer-reviewed journals and scientific conferences. Ethical approval is not applicable for this systematic review since no primary data were collected. Instead, the one used was publicly available or published in scientific journals.

\section{Author affiliations}

${ }^{1}$ Department of Bioethics and Health Professionalism, Muhimbili University of Health and Allied Sciences, Dar es Salaam, United Republic of Tanzania 
${ }^{2}$ Department of Community Health, Muhimbili University of Health and Allied Sciences, Dar es Salaam, United Republic of Tanzania

${ }^{3}$ Department of Clinical Pharmacology, Muhimbili University of Health and Allied Sciences, Dar es Salaam, United Republic of Tanzania

${ }^{4}$ Department of Nursing Management, Muhimbili University of Health and Allied Sciences, Dar es Salaam, United Republic of Tanzania

${ }^{5}$ Department of Paediatric, Kilimanjaro Christian Medical College, Kilimanjaro, United Republic of Tanzania

Collaborators Transforming Health Professions Education in Tanzania (THET) Project, Alex Mremi, Dorah Mrema, Honest Massawe, Nicholas Bartholomeo, Eva Malindisa, Matiko Mwita, James Mundamshimu

Contributors GP and BFS conceptualised and developed the systematic review protocol. GP drafted the manuscript. BM, BFS, TM, BM and MA revised and provided substantive feedback on the manuscript. All authors have read and approved the final manuscript.

Funding The systematic review is funded by Fogarty International Centre (FIC) of the National Institutes of Health under award number 1R25TW011227-01.

Disclaimer The funder has no role in developing the protocol. The content is solely the responsibility of the authors and does not necessarily represent the official views of the US National Institutes of Health (NIH).

Competing interests None declared.

Patient and public involvement Patients and/or the public were not involved in the design, or conduct, or reporting, or dissemination plans of this research.

Patient consent for publication Not required.

Provenance and peer review Not commissioned; externally peer reviewed.

Open access This is an open access article distributed in accordance with the Creative Commons Attribution Non Commercial (CC BY-NC 4.0) license, which permits others to distribute, remix, adapt, build upon this work non-commercially, and license their derivative works on different terms, provided the original work is properly cited, appropriate credit is given, any changes made indicated, and the use is non-commercial. See: http://creativecommons.org/licenses/by-nc/4.0/.

ORCID iD

Godwin Pancras http://orcid.org/0000-0002-7802-9490

\section{REFERENCES}

1 Cox LE, Rouff JR, Svendsen KH, et al. Community Advisory boards: their role in AIDS clinical trials. Health Soc Work 1998;23:290-7.

2 Shubis K, Juma O, Sharifu R, et al. Challenges of establishing a community Advisory board (cab) in a low-income, low-resource setting: experiences from Bagamoyo, Tanzania. Health Res Policy Syst 2009;7:16.

3 Morin SF, Maiorana A, Koester KA, et al. Community consultation in HIV prevention research: a study of community Advisory boards at 6 research sites. J Acquir Immune Defic Syndr 2003;33:513-20.

4 Lawrence $\mathrm{C}$, Stewart $\mathrm{K}$. The challenge of community representation: lessons from six HIV clinical research community Advisory boards in Uganda. J Empir Res Hum Res Ethics 2016;11:311-21.

5 Reddy P, Buchanan D, Sifunda S, et al. The role of community Advisory boards in health research: divergent views in the South African experience. Sahara J 2010;7:2-8.

6 NIMH Multisite HIV/STD Prevention Trial for African American Couples Group. The role of community Advisory boards (CABs) in project Eban. J Acquir Immune Defic Syndr 2008;49:S68-74.

7 Morin SF, Morfit S, Maiorana A, et al. Building community partnerships: case studies of community Advisory boards at research sites in Peru, Zimbabwe, and Thailand. Clin Trials 2008:5:147-56

8 U.S. Department of Health \& Human Services. The Belmont Report: Ethical Principles and Guidelines for the Protection of Human Subjects of Research [Internet]. USA: The National Commission for the Protection of Human Subjects of Biomedical and Behavioral Research, 1979. https://www.hhs.gov/ohrp/regulations-and-policy/ belmont-report/read-the-belmont-report/index.html

9 Emanuel EJ, Wendler D, Grady C. What makes clinical research ethical? JAMA 2000;283:2701-11.

10 Thomas J, Brunton J. EPPI-Reviewer 4: software for research synthesis EPPI-Cent Softw Lond soc SCI Res unit UCL insT Educ, 2010.

11 Moher D, Liberati A, Tetzlaff J, et al. Preferred reporting items for systematic reviews and meta-analyses: the PRISMA statement. PLoS Med 2009;6:e1000097.

12 CASP. Critical Appraisal Skills Programme [Internet]. CASP Qualitative checklist, 2018. Available: https://casp-uk.net/casp-toolschecklists/

13 Lockwood C, Munn Z, Porritt K. Qualitative research synthesis: methodological guidance for systematic reviewers utilizing metaaggregation. Int J Evid Based Healthc 2015;13:179-87.

14 Dixon-Woods M, Cavers D, Agarwal S, et al. Conducting a critical interpretive synthesis of the literature on access to healthcare by vulnerable groups. BMC Med Res Methodol 2006;6:35.

15 Thomas J, Harden A. Methods for the thematic synthesis of qualitative research in systematic reviews. BMC Med Res Methodol 2008;8:45.

16 International ethical guidelines for health-related research involving humans. Council for International Organizations of Medical Sciences(ClOMS). Available: https://cioms.ch/shop/product/ international-ethical-guidelines-for-health-related-research-involvinghumans/

17 World Medical Association. World Medical association Declaration of Helsinki: ethical principles for medical research involving human subjects. JAMA 2013;310:2191-4. 